Conclusion Rates of these four risky behaviours were high and, with the exception of binge drinking, were largely behaviours adopted in the USA Newly arrived migrant men are a group at high risk for sex and drug related STI/HIV. Interventions to prevent transmission in this vulnerable, difficult-to-access and highly mobile population are greatly needed.

Abstract P1-S2.75 Table 1 Behaviours in sending country and in New Orleans $(\mathrm{N}=93)$

\begin{tabular}{llclc}
\hline Behaviours & $\begin{array}{l}\text { Patronage } \\
\text { of FSW }\end{array}$ & MSM & Binge* & Crack cocaine \\
\hline $\begin{array}{l}\text { Reported in } \\
\text { sending country }\end{array}$ & $16 / 93(17.2 \%)$ & $3 / 93(3.2 \%)$ & $39 / 91(42.9 \%)$ & $2 / 93(2.2 \%)$ \\
$\begin{array}{l}\text { Reported in } \\
\text { New Orleans }\end{array}$ & $60 / 93(64.5 \%)$ & $10 / 93(10.8 \%)$ & $35 / 91(38.5 \%)$ & $12 / 93(12.9 \%)$ \\
$\begin{array}{l}\text { Not reported in } \\
\text { sending country } \\
\text { (of those reported } \\
\text { in New Orleans) }\end{array}$ & $50 / 60(83.3 \%)$ & $8 / 10(80.0 \%)$ & $15 / 35(42.9 \%)$ & $12 / 12(100.0 \%)$ \\
\hline
\end{tabular}

*2 men had missing information.

\section{P1-S2.76 SEXUAL CONCURRENCY AMONG LATINO MIGRANT MEN IN NEW ORLEANS}

doi:10.1136/sextrans-2011-050108.133

P Kissinger, C Anderson-Smits, S Kovacs, 0 Salinas, J H, N Schmidt. Tulane University, School of Public Health and Tropical Medicine, New Orleans, USA

Background Sexual concurrency and migration have both been implicated in the spread of HIV/STI. Migrant men are highly mobile, but their level of sexual concurrency has not been well studied. The purpose of this study was to examine the prevalence of and factors associated with sexual concurrency and to examine condom use within concurrent relationships among a group of newly arrived Latino migrant men (LMM) in New Orleans.

Methods LMM enrolled in an ongoing cohort study, who had at least one female sexual partner during follow-up were interviewed a three time points over 6 months. Concurrency was calculated by asking start and stop date of each sexual relationship as well as intention to continue. Partnerships that had overlapping dates were considered concurrent. The association between selected individual and environmental factors and sexual concurrency was examined using generalised estimated equations (GEE).

Results At baseline, LMM ( $\mathrm{n}=90)$ were mostly Honduran (77.9\%), employed $(80.0 \%)$, worked in construction (55.6\%), were uncircumcised (88.9\%), were living with family (51.1\%) and did not have a main sex partner (74.4\%). Their mean age was 35.4 (SD 10.6) and they had been in New Orleans for average of 4.70 years (SD 0.89). During follow-up, 30 (33.3\%) had at least one concurrent relationships, 10 (11.1\%) had only concurrent relationships, and 59 (65.6\%) had no concurrent relationships. In 239 observations, sexual partnering and consistent condom use was: concurrent (18.0\%/53.5\%), multiple but non-concurrent partners (5.9\%/78.6\%), and monogamous (50.6\%/30.8\%), while $25.5 \%$ were abstinent. Factors associated with sexual concurrency included: younger age, drug use, and living in crowed housing while belonging to organizations or sport team was protective. Of these 43 concurrent events, $21.0 \%$ included a risky partner (ie, female sex worker or casual partner) and main partner and $65.2 \%$ had at least one non-Latina partner. Of the 29 concurrent relationships that included a FSW, 5 (17.2\%) did not use a condom with the sex worker.

Conclusion This sample of LMM exhibited high rates of concurrency with a potential for bridging. Drug prevention and interventions that promote social connectedness are needed to reduce concurrency among this mobile group.

\section{Epidemiology poster session 3: Burden of disease}

\section{P1-S3.01 TRENDS IN THE INCIDENCE OF HOSPITALISATION FOR CHLAMYDIA-RELATED SEOUELAE AMONG WOMEN}

doi:10.1136/sextrans-2011-050108.134

${ }^{1} \mathrm{~B}$ Liu, ${ }^{1} \mathrm{~B}$ Donovan, ${ }^{1} \mathrm{H}$ Wand, ${ }^{1} \mathrm{R}$ Guy, ${ }^{2} \mathrm{~J}$ Hocking, ${ }^{1} \mathrm{~J}$ Kaldor. ${ }^{1}$ National Centre in HIV Epidemiology and Clinical Research, Coogee, Australia; ${ }^{2}$ University of Melbourne, Australia

Background Chlamydia infection is thought to increase the risk of infertility and ectopic pregnancy. Over the last 10 years in Australia, chlamydia testing and notifications have increased dramatically, particularly in young women. As it is unclear how much increasing notifications reflect a true increase in chlamydia incidence, we sought to investigate rates of hospitalisation for the chlamydiarelated sequelae, infertility and ectopic pregnancy in New South Wales (NSW), Australia's largest state with a population of 7 million.

Methods Routine data on hospitalisations for infertility and ectopic pregnancy, perinatal data, and population census data from 2001 to 2008 were used to estimate annual age-specific hospitalisation rates for infertility and ectopic pregnancy in women aged 15 to 44 years for the entire state of New South Wales. Hospital separations occuring within each year belonging to the same woman were linked using probabilistic linkage of identifiers so that multiple admissions by one woman were only counted once.

Results From 2001 to 2008 the number of women hospitalised for infertility increased from 7050 to 9978 . The greatest increase was seen in women aged 35 to 44 years, from 6.4 [95\% CI 6.2 to 6.6$]$ to 11.2 [10.9 to 11.5 ] per 1000 population ( $p$ trend $<0.001$ ). There was no significant change among women aged 15 to 24 years (ptrend=0.3); see Abstract P1-S3.01 figure 1A. For women with
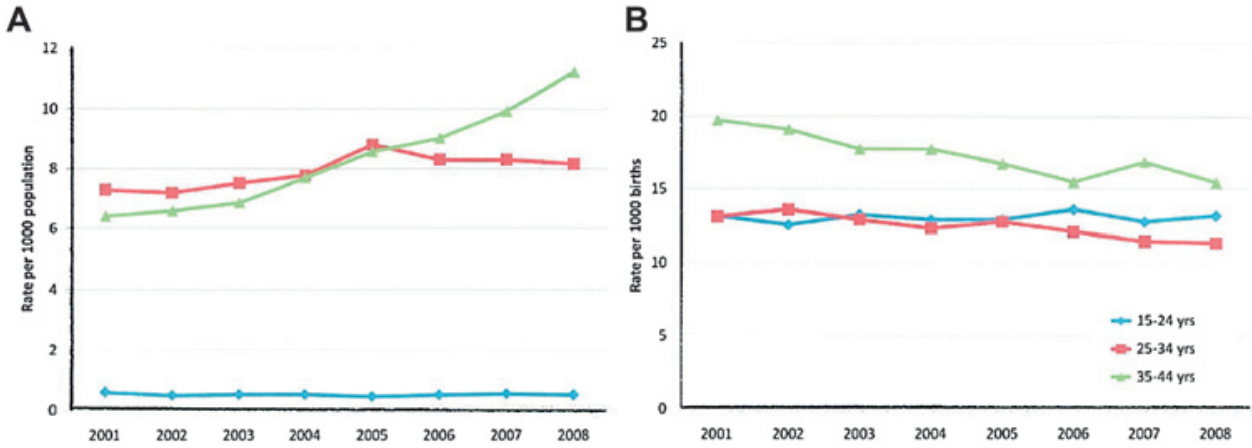

Abstract P1-S3.01 Figure 1 Women hospitalised for A) infertility and B) ectopic pregnancy in NSW, 2001 to 2008. 
ectopic pregnancy overall the number of women hospitalised decreased from 1204 to 1192, a decrease in annual rates from 14.3 [13.5 to 15.1] to 12.6 [11.9 to 13.3] per 1000 births ( $p$ trend $<0.001$ ) but this decrease was mostly in women aged between 25 to 44 years. There was no appreciable fall in hospitalisations for ectopic pregnancy in women aged 15 to 24 years (13.1 [11.5 to 15.0 ] to 13.1 [11.4 to 15.0] per 1000 births; ptrend=0.9; see Abstract P1-S3.01 figure $1 \mathrm{~B})$.

Conclusions Among women aged 25 to 44 years decreases in hospitalisations for ectopic pregnancy and increases in hospitalisations for infertility were observed. The relationships between chlamydia prevalence, testing and notifications do not appear to be straightforward.

\section{P1-S3.02 THE ECONOMIC BURDEN OF CHLAMYDIA AND GONORRHOEA IN CANADA}

doi:10.1136/sextrans-2011-050108.135

L Smylie, P Lau, R Lerch, C Kennedy, R Bennett, B Clarke, A Diener. Public Health Agency of Canada, Ottawa, Canada

Background The latest estimate of the economic burden of chlamydia and gonorrhoea in Canada was modelled with data from 1990 and was published in 1992 by Ron Goeree and Paul Gully. Given the changes that have occurred since in diagnostic testing technology, the availability of new data sources and increasing rates of the two infections, a new model using updated data from 2000 is called for.

Methods Diagnostic test costs were estimated using provincial laboratory data on the number of diagnostic tests performed for Chlamydia trachomatis (CT) and Neisseria gonorrhoeae (GC) for the year 2000. Direct costs of CT and GC from drugs, hospitals, and physician billings were estimated using the Economic Burden of Illness in Canada (EBIC) data. Direct costs of associated sequelae of each infectious disease were also included in the model. Indirect costs estimated in the model included production losses from both infectious diseases and their associated sequelae. Sensitivity analyses were conducted to provide upper, base and lower-bound estimates of the total cost.

Results The preliminary combined estimate for both direct and indirect costs of CT and GC (in 2000 dollars) ranges from approximately $\$ 31.5$ to $\$ 178.4$ million (CAD).

Conclusions Further work is required to improve data access and estimates of the burden of infection in Canada. A top priority should be to improve the data infrastructure by expanding data linkages within and among provinces around laboratory tests. The majority of costs related to CT and GC are attributable to drug, hospital and physician costs, suggesting that much of the burden of these two infections can be reduced through implementation of effective prevention programs. The number of CT infections has increased exponentially since 2000, warranting further modelling considering current incidence rates and inflation costs.

\section{P1-S3.03 MORTALITY AMONG MEN WHO HAVE SEX WITH MEN SEEN IN STD CLINIC PATIENTS: HIGH RATES OF SUICIDE AND LOW RATES OF ANAL CANCER DEATH}

doi:10.1136/sextrans-2011-050108.136

${ }^{1} \mathrm{~S}$ Glick, ${ }^{2} \mathrm{R}$ Kerani, ${ }^{2} \mathrm{M}$ Golden. ${ }^{1}$ University of Washington, Seattle, USA; ${ }^{2}$ Public Health, Seattle and King County, Seattle, USA

Background Because men who have sex with men (MSM) are not identifiable at a population level, there are few data on cause-specific mortality rates among MSM. MSM are more likely to report suicide attempts than heterosexuals, but it is not known if suicide mortality is higher among MSM. Likewise, although anal cancer is more common among MSM than among heterosexuals, the absolute risk of anal cancer death among MSM is not known. We compared suicide and anal cancer mortality rates among MSM and heterosexual males and females in an STD clinic population.

Methods We enumerated all Public Health-Seattle and King County STD Clinic attendees from 1993-2007 $(n=86,807)$. Using 1993-2008 mortality data from Washington State $(n=702850)$, we identified STD clinic attendees who had died and their underlying cause of death. We calculated suicide and anal cancer mortality rates for MSM, heterosexual men, and women, and standardised these rates using the age, race, and gender distribution of King County residents (US Census, 2000). We calculated adjusted incidence rate ratios (IRR) among STD clinic attendees using Poisson regression. To compare mortality rates among STD clinic attendees to the general population, we calculated rates in King County.

Results Among STD clinic attendees, 14\% were MSM, 52\% were heterosexual men, and 34\% were women. For the period between 1993 and 2008, we matched 1901 Washington state deaths to STD clinic attendees for an overall unadjusted mortality rate of 1.8 deaths per 1000 person-years. The standardised suicide mortality rate among MSM was 0.20 per 1000 person-years, compared with 0.11 among heterosexual men and 0.09 among all women. Adjusted for age and race, among STD clinic attendees, MSM were 1.6 times more likely to die from suicide than heterosexual men (IRRadj=1.6, $95 \%$ CI 1.02 to 2.61 ) and nearly four times more likely than women (IRRadj=3.9, 95\% CI 2.14 to 7.24 . The suicide mortality rate among MSM in the STD clinic was higher than the rate among all men in King County (standardised rate ratio $=1.5$ ), adjusted for age and race. We identified no deaths due to anal cancer in any subpopulation (95\% CI 0 to 0.4 per 100000 person-years).

Conclusions These data suggest that, at least among STD clinic attendees, MSM are approximately twice as likely to die from suicide as heterosexual men, and these rates are higher than the general population. Anal cancer death rates among King County MSM appear to be low.

\section{P1-S3.04 DEATH IN THE ERA OF HAART: NOT ALL ABOUT AIDS}

doi:10.1136/sextrans-2011-050108.137

R Smith, M Kall, V Delpech. Health Protection Agency, London, UK

Background Mortality from AIDS has dramatically reduced since the introduction of effective antiretroviral therapy, Nevertheless, HIV infected individuals remain at higher risk of early death. We investigate all causes of deaths among HIV diagnosed adults reported nationally in the HAART era.

Methods Analyses of data from national HIV surveillance linked to the Office National Statistics. Deaths among all adults $(\geq 15)$ diagnosed between 1997 and 2008 in England and Wales (E\&W) were reviewed and grouped by cause of death (COD) and risk information. Death rates were calculated using a census of all persons accessing HIV care in 2008 (SOPHID).

Results The crude mortality rate among HIV-infected individuals (15-59yrs) fell from 21.8 to 8.2 per 1000 persons living with diagnosed HIV in 2008. A total of 3640 (5.6\%) persons diagnosed over the 12-year period were reported to have died [3544 had a cause of death]. The overall median age at death was 41 yearss [IOR: $35-50$ years]. AIDS related mortality accounted for $56 \%$ of all deaths: $51 \%$ among MSM, 61\% among heterosexuals and $34 \%$ among IDUs. AIDS-related respiratory infection (including PCP) was the most common COD in MSM and heterosexuals whereas 